INRA Prod. Anim.,

2013, 26 (3), 289-300

\title{
La production de viande de chameau : état des connaissances, situation actuelle et perspectives
}

\author{
B. FAYE ${ }^{1,2,3,4}$, O. ABDELHADI 2,3,4,5, G. RAIYMBEK ${ }^{6}$, I. KADIM7 , J.-F. HOCQUETTE ${ }^{8,9}$ \\ ${ }^{1}$ Camel Project UTF/SAU/044/SAU, BP n ${ }^{\circ} 761,11942$ Kharj, Arabie Saoudite \\ ${ }^{2}$ CIRAD, UMR0868 SELMET, Campus International de Baillarguet, TA C/112A, \\ F-34398 Montpellier, France \\ ${ }^{3}$ INRA, UMR0868 SELMET, 2 place Viala, F-34060 Montpellier, France \\ ${ }^{4}$ SupAgro, UMR0868 SELMET, 2 place Viala, F-34060 Montpellier, France \\ 5 University of Kordofan, Faculty of Natural Resources and Environmental Studies, \\ Department of Animal Production, P.O. Box 716, Khartoum, Sudan \\ ${ }^{6}$ Faculté de Biologie, Université Al-Farabi, 71 avenue Al-Farabi, 050040, Almaty, Kazakhstan \\ ${ }^{7}$ Department of Animal and Veterinary Sciences, College of Agricultural and Marine Sciences, \\ Sultan Qaboos University, P.O. Box 34 Al-Khoud, Muscat, Oman \\ 8 INRA, UMR1213 Herbivores, F-63122 Saint-Genès-Champanelle, France \\ ${ }^{9}$ Clermont Université VetAgro Sup, UMR1213 Herbivores, BP 10448, F-63000 Clermont-Ferrand, France \\ Courriel : bernard.faye@cirad.fr
}

Bien que marginale (moins de $1 \%$ du marché des viandes rouges), la viande de chameau fait l'objet d'un intérêt grandissant auprès des consommateurs des pays arides, tant du point de vue économique que diététique. S'appuyant sur les méthodologies d'étude mises au point pour d'autres espèces, les connaissances sur cette viande s'améliorent grandement permettant une meilleure appréciation de son intérêt pour les acteurs de la filière.

La viande de chameau ${ }^{1}$ est régulièrement consommée dans les pays arides où dromadaires et chameaux de Bactriane représentent une part non négligeable du cheptel local. Cependant, le grand public est souvent surpris d'apprendre que le chameau «ça se mange ", tout comme son lait se boit. Les recherches sur la viande dans cette espèce, les études sur le marché qu'elle représente, les travaux sur les procédés de transformation sont assez récents, ce qui explique finalement le peu d'informations dont on dispose a priori sur ce produit. Cependant, aujourd'hui dans un certain nombre de pays d'élevage, l'intérêt pour la viande des grands camélidés est grandissant comme en témoigne l'augmentation du nombre de publications qui lui sont consacrées. Une récente synthèse dans la revue "Meat Science » est disponible (Kadim et al 2008), mais elle n'aborde pas les questions liées au marché de la viande et au potentiel de production des systèmes d'élevage. De plus, la récente publication de l'ouvrage collectif «Camel meat and meat products » (Kadim et al 2013a) a apporté des éléments complémentaires qui nous ont semblé judicieux de synthétiser ici.

Dans la présente synthèse, seront donc exposées, outre les connaissances acquises sur la viande de chameau, au regard des travaux les plus récents, la situation actuelle du marché de ce produit et les perspectives de développement au travers de quelques projets en cours de réalisation. La viande des petits camélidés (lama et alpaga essentiellement), pourtant appréciée des consommateurs des pays andins, ne sera pas abordée ici, d'une part parce qu'on ne dispose que de peu d'informations sur le sujet, d'autre part, parce qu'elle relève d'un tout autre marché que la viande des grands camélidés.

\section{1 / Le potentiel de produc- tion de viande caméline}

Le volume de viande caméline disponible sur le marché est bien évidemment dépendant des effectifs à l'échelle mondiale. Or, il est difficile de connaître exactement la taille de la population caméline mondiale. Animal des pasteurs, voire des nomades dans la plupart des cas, la population caméline est soumise à une importante mobilité, ce qui rend son accessibilité difficile lors des procédures de recensement. De plus, il n'existe pas de campagne de vaccination obligatoire comme pour le cheptel bovin, ce qui exclut ces animaux des statistiques des services vétérinaires. Dans la plupart des cas, la population caméline est donc estimée, plutôt que recensée. Officiellement, le nombre de camélidés dans le monde est estimé selon la FAO à environ 25 millions de

\footnotetext{
1 « Chameau » est un terme générique qui comprend le « chameau dromadaire à une bosse » et le « chameau de Bactriane à 2 bosses ». Dans la suite du texte, ils seront spécifiés sous l'appellation « dromadaire » et « Bactriane », l'appellation « chameau » couvrant les deux espèces.
} 
Figure 1. Comparaison des effectifs d'herbivores domestiques à l'échelle mondiale (FAOSTAT 2011).

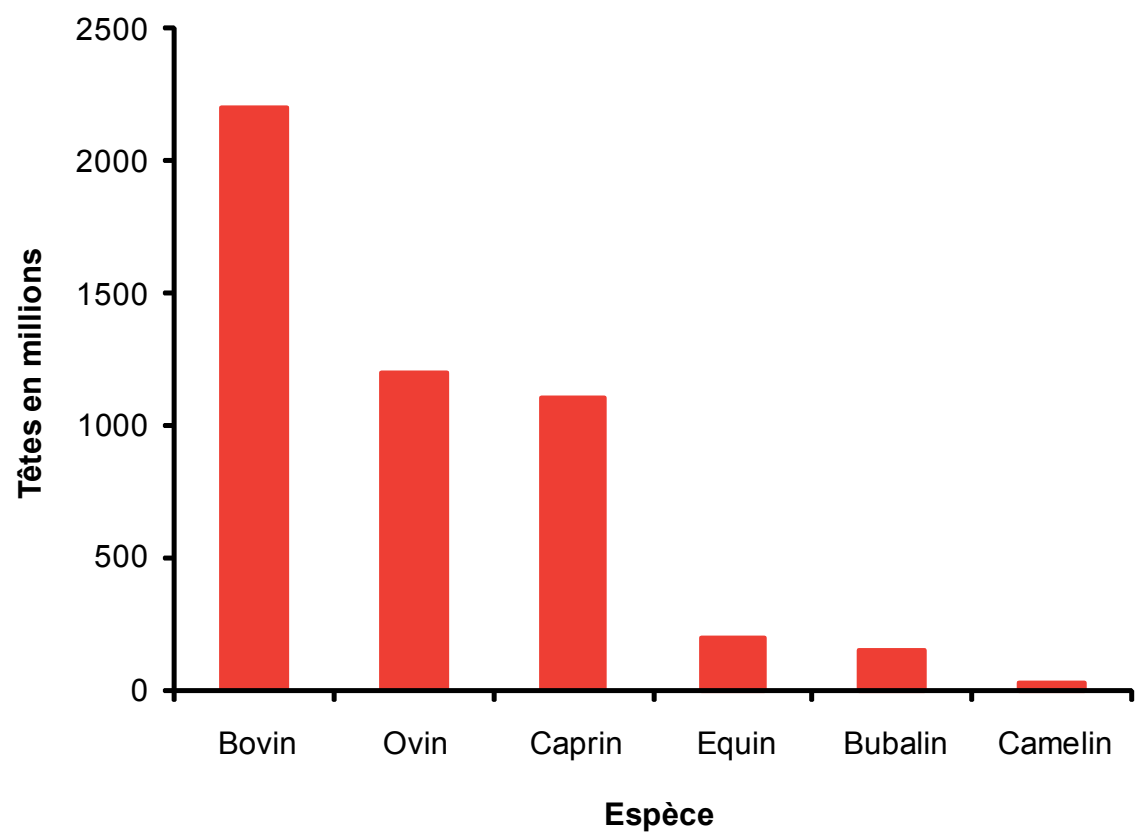

têtes (FAOSTAT 2011). Ce nombre est probablement sous-estimé, tout particulièrement dans les pays sahéliens (Mauritanie, Mali, Niger, Tchad, Soudan, Ethiopie) où le nombre de têtes a été réajusté après des recensements exhaustifs comme par exemple au Tchad où les effectifs sont passés de 800000 à 1,3 millions de têtes en peu d'années (Faye et Bonnet 2012). Par ailleurs, les chiffres de la FAO ne prennent pas en compte les dromadaires ensauvagés d'Australie qui selon diverses estimations seraient au nombre de 1 à 1,5 millions de têtes (Ward et al 2010). Si on se base sur les estimations nationales, parfois éloignées des chiffres avancés par la FAO (par exemple en Arabie Saoudite, les chiffres varient entre 280000 et 800000 têtes selon les sources), on peut estimer la population mondiale de grands camélidés à 30 millions de têtes dont $95 \%$ de dromadaires et $5 \%$ de chameaux de Bactriane, soit toutefois moins de $1 \%$ de la population des herbivores domestiques (figure 1).

Près de $60 \%$ de la population cameline est concentrée dans les pays de la Corne de l'Afrique (Somalie, Soudan, Ethiopie, Kenya, Erythrée, Djibouti). La population mondiale augmente régulièrement avec une croissance annuelle de $3,4 \%$. Depuis 1961, selon les statistiques de la FAO, le nombre de grands camélidés a plus que doublé. Cependant, on observe des patrons de croissance différents selon les pays. Globalement, on peut distinguer 5 types de pays (figure 2) :

- Type A : pays présentant une croissance rapide, mais récente (Algérie, Tchad, Mali, Mauritanie, Oman, Qatar, Syrie, Yémen, Ethiopie, Erythrée) ;
- Type C : pays présentant un effectif camelin stable puis un léger déclin (Liban, Libye et Sénégal);
Type D: pays marqués par une population en déclin régulier (Afghanistan, Chine, Inde, Israël, Jordanie, Mongolie, républiques d'Asie Centrale, Russie) ;

- Type E : pays face à un déclin important, mais irrégulier (Irak, Maroc -hors zone saharienne, Turquie).

On pourrait ajouter à cette liste, les pays d'introduction de circonstances, soit parce qu'il s'agit de pays arides ayant une écologie où le dromadaire peut s'adapter facilement (par exemple, la Namibie a déclaré officiellement à la FAO, en 2011, sa population de dromadaires introduite dans le désert du Kalahari), soit parce que l'espèce contribue à la diversification de l'élevage, notamment en Europe et en particulier en France (Faye et al 1995), en général pour le loisir, mais parfois aussi pour la production (il existe un élevage de chamelles laitières en Hollande notamment, Smits et Montety 2009). Dans cette catégorie de pays, l'Australie joue un rôle à part, car la quasi-totalité du cheptel camelin est maintenant retourné à l'état sauvage (Faye et al 2002), et la pression exercée par une population abondante constitue un problème environnemental croissant que les autorités tentent de résoudre par diverses voies, y compris la destruction en masse des camélidés (Saalfeld et Edward 2010). L'une des pistes proposées est justement la valorisation de la viande d'abattage sur les marchés à l'export sur lesquels nous reviendrons plus loin.

Figure 2. Types de croissance démographique des cheptels camelins nationaux entre 1961 et 2010 (calculé d'après FAOSTAT 2011).

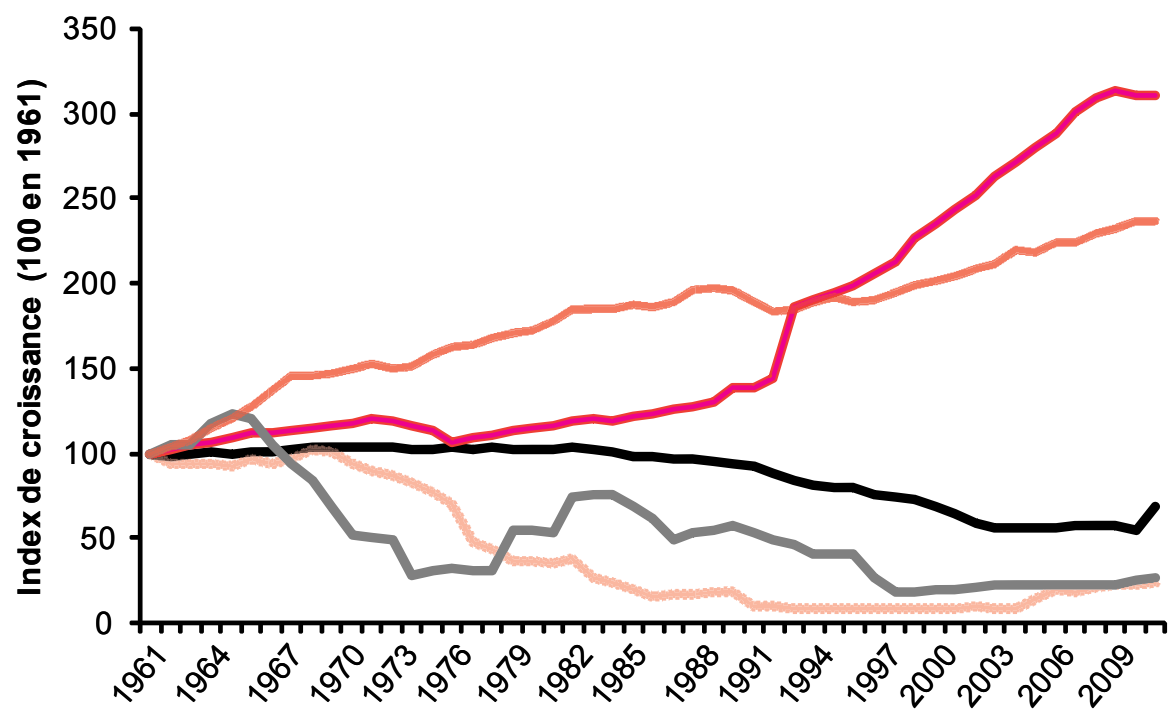

Années

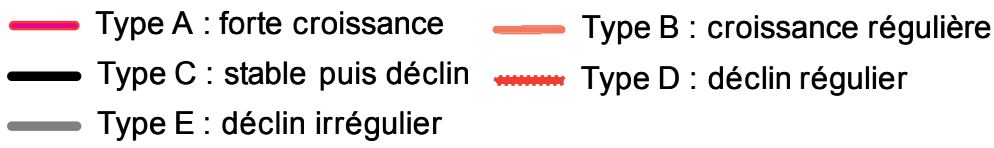




\section{2 / Le marché de la viande de chameau}

Les grands camélidés sont le type même d'animaux multi-usages : lait, viande, laine, transport, course, tourisme, travaux agricoles et « concours de beauté $»^{2}$. Il n'y a pas d'autres animaux domestiques capables de rassembler autant de services pour l'Homme. La viande est la seule production requérant l'abattage de l'animal. En conséquence, la production de viande est associée à une gestion raisonnée des troupeaux en termes de sélection des animaux destinés à l'abattage (en particulier les jeunes mâles non destinés à la reproduction où les femelles de réforme) ou d'organisation des marchés au niveau local ou régional.

Figure 3. Evolution du taux d'abattage des grands camélidés à l'échelle mondiale depuis 1961 (FAOSTAT 2011).

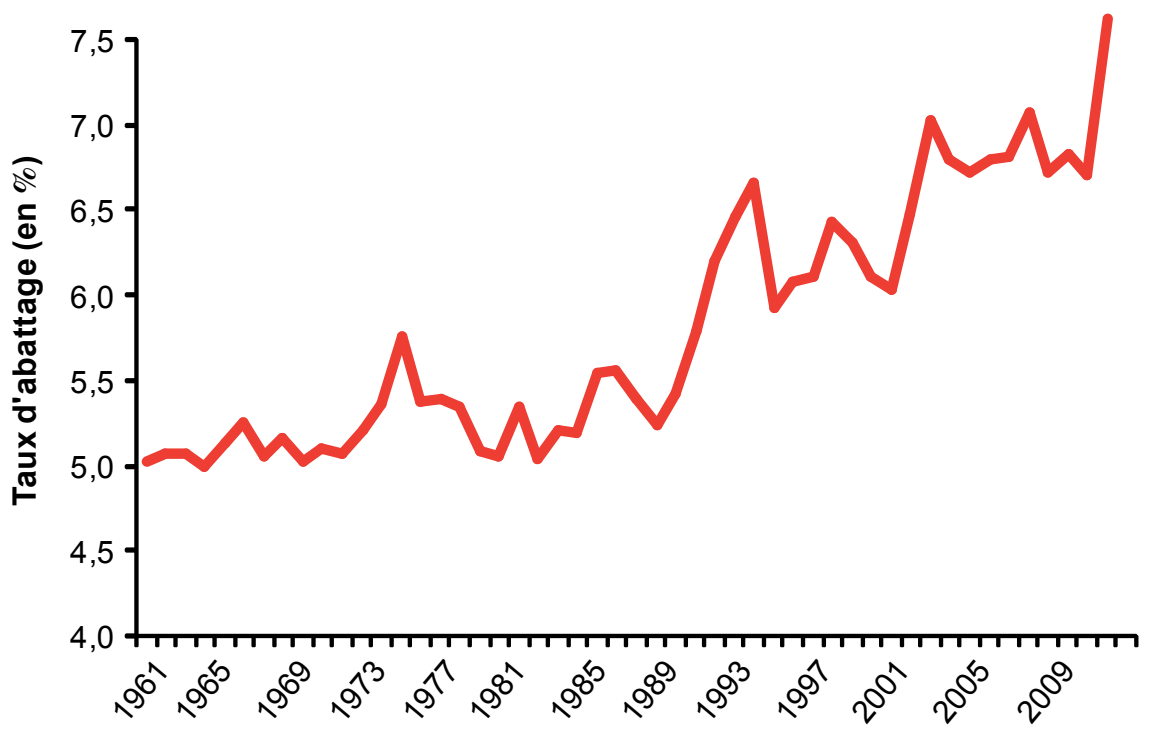

Année

Figure 4. Pyramide des âges (en années) à l'abattage (abattoir de Laâyoune, Maroc).

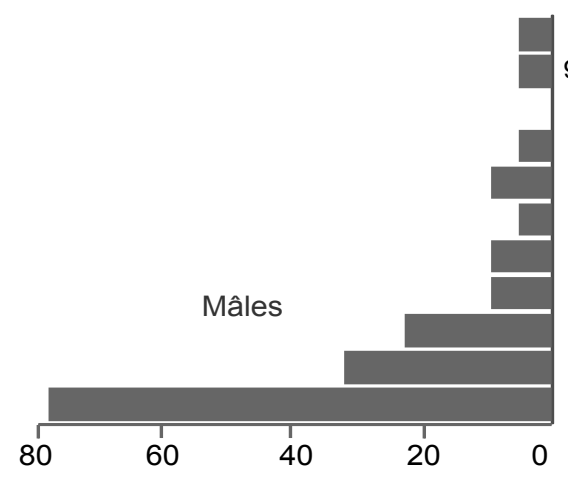

Nombre d'animaux abattus en 2 mois
Il est à noter également, que le marché mondial de la viande de chameau est étroitement lié (mais non exclusivement si on y intègre le chameau de Bactriane) au monde arabo-musulman (Faye et Porphyre 2011) et qu'en conséquence, la viande disponible sur les marchés doit être Halal (Mbaga 2013). Cela limite de fait les flux d'animaux en provenance des pays ne pouvant délivrer de certification Halal (cela vise en particulier les pays comme l'Inde ou l'Australie).

Il convient d'ajouter aussi que la consommation de viande de chameau fait l'objet de tabous ou du moins de réticences à la consommation dans certaines sociétés. Manger du chameau est prohibé pour les juifs pratiquants. L'abattage n'est pas autorisé chez les
Raikas en Inde et les chrétiens coptes d'Ethiopie (Bekhit et Farouk 2013). Plus généralement, bien que licite, la consommation de chameau dans les sociétés pastorales plaçant cette espèce au sommet de la hiérarchie animale, n'est pas appréciée par la communauté, comme c'est le cas chez les touaregs (Cabalion 2013).

Les seules statistiques disponibles (FAOSTAT 2011) sur la viande de chameau concernent le nombre d'animaux abattus, le poids moyen des carcasses et la production totale de viande. On ne dispose pas, en revanche, de statistiques globales sur le type d'animaux abattus ou le traitement des produits carnés. Un nombre significatif d'animaux est abattu en dehors des circuits officiels, expliquant probablement une sous-estimation de la production.

\section{1 / Le taux d'abattage}

Le pourcentage de chameaux officiellement abattus (rapporté à la population totale) à l'échelle mondiale a régulièrement augmenté depuis les années 1960 passant de 5 à $7 \%$ (figure 3 ). Cet accroissement pourrait être expliqué par une meilleure organisation de la filière et par une diminution des abattages nonofficiels.

Ce pourcentage est évidemment plus élevé chez les mâles que chez les femelles, surtout dans les systèmes laitiers, mais seules quelques statistiques locales sont disponibles. Par exemple, après 2 mois de suivi à l'abattoir de Laâyoune (Sud-Maroc, figure 4) où une quinzaine d'animaux sont abattus en moyenne chaque jour, on a pu observer que $44 \%$ des mâles abattus avaient moins de un an, vs 14\% pour les femelles (Faye 2013). Les femelles de réforme représentaient $28 \%$ des femelles abattues vs 7,7\% seulement pour les mâles réformés (figure 4).

Le taux d'abattage annuel est cependant très variable d'un pays à l'autre (figure 5). Il est en moyenne plus faible en Afrique (5,7\%) qu'en Asie (7,6\%). $\mathrm{Au}$ niveau régional, il apparaît particulièrement élevé au Proche-Orient $(32,6 \%)$, comparé à l'Extrême-Orient $(18,0 \%)$, à l'Afrique du Nord $(26,3 \%)$ et à l'Afrique de 1 'Ouest $(9,1 \%)$. Dans les autres régions, l'importance de l'abattage semble bien moins marquée : Afrique de l'Est (4,9\%), Asie Centrale $(2,0 \%)$ et Asie du Sud (2,8\%). Ce taux est faible, soit pour des raisons religieuses (cas de l'Inde) soit parce que la priorité est donnée à la production laitière (cas de l'Afrique de l'Est et de l'Asie Centrale).

\footnotetext{
2 On ne peut pas vraiment comparer ces manifestations à des concours agricoles où les plus beaux animaux sont en compétition sur la base de leurs performances zootechniques (poids, production laitière), mais plutôt aux concours canins ou félins, où sont jugés des morphologies selon des critères stricts.
} 
Figure 5. Taux d'abattage (pourcentage d'animaux abattus par rapport à la population totale) dans la population caméline en 2011 (source FAOSTAT 2011, Faye et Bonnet 2012).

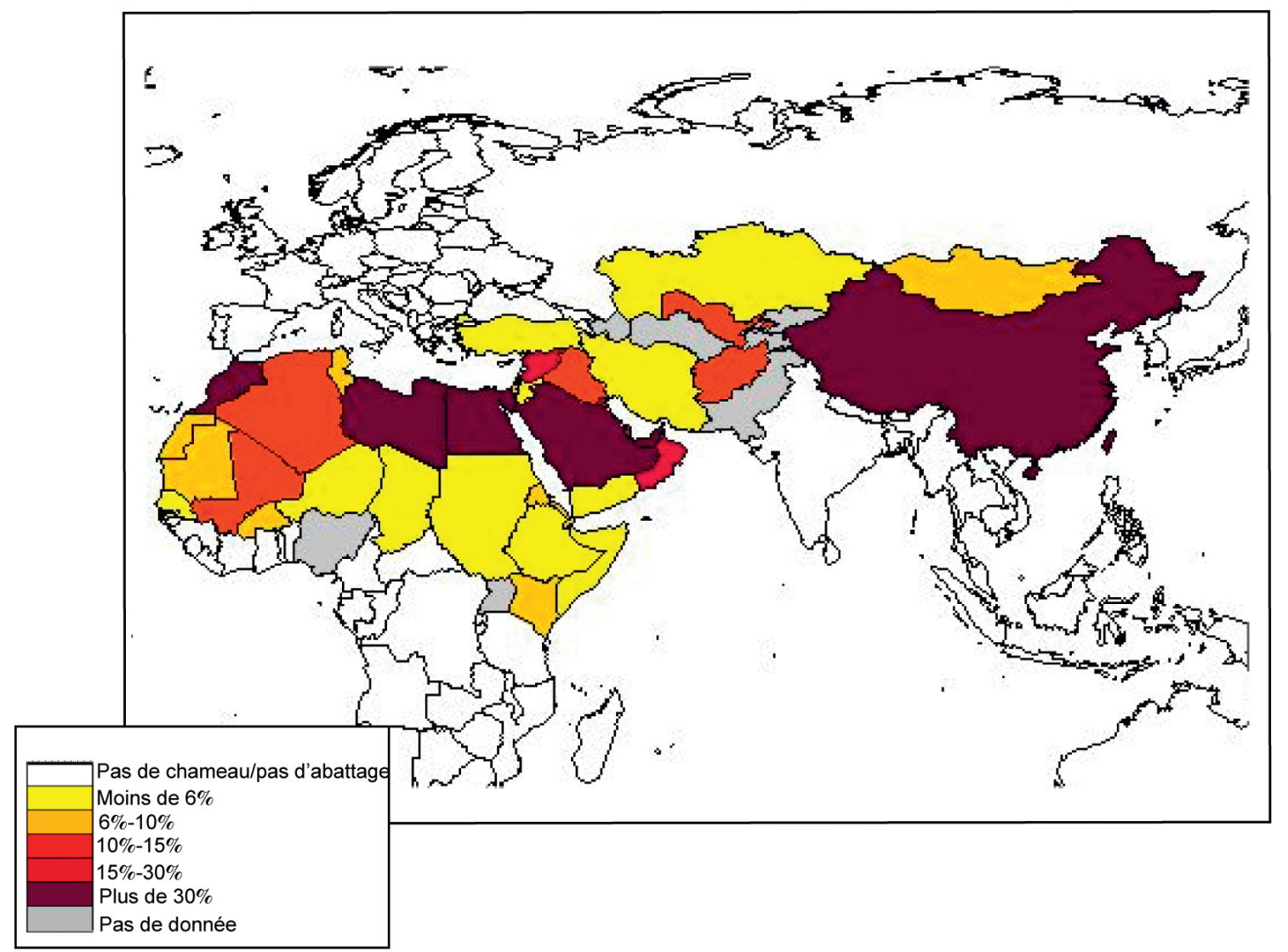

A l'échelle des pays, le taux d'abattage varie de 0 à $118 \%$. Bien évidemment, les pays présentant des taux élevés, voire supérieurs à la population caméline nationale (plus de $100 \%$ !) correspondent aux pays importateurs d'animaux sur pied pour satisfaire la demande locale en viande caméline. C'est le cas de l'Afrique du Nord (Libye et Egypte surtout) et surtout de la Péninsule Arabique comme on le verra plus loin. Le cas de la Chine est à part, car le taux d'abattage élevé (plus de $30 \%$ ) est plutôt lié à un important déstockage qui explique le déclin de la population caméline depuis une décennie. A contrario, les taux d'abattage les plus faibles sont observés dans les pays exportant des animaux sur pied, essentiellement dans la zone sahélienne (source FAOSTAT 2011). Il faut rappeler cependant qu'il s'agit d'abattages déclarés et donc probablement sous-estimés.

\section{2 / Contribution à la production de viande}

Depuis 1961, la croissance de la production mondiale de viande de chameau a été régulière à un rythme annuel de 2,8\% passant de 123000 à 381000 tonnes. Néanmoins, la contribution du chameau à la production mondiale de viande rouge est très marginale, du fait de la taille de la population caméline : la viande de chameau représente $0,13 \%$ de la viande produite dans le monde et $0,45 \%$ de la viande rouge (Faye 2013). Cependant, les grands camélidés étant confinés dans les pays arides, leur contribution apparaît plus élevée localement. En Afrique de l'Est, la viande de dromadaire représente $4,1 \%$ de la viande rouge produite, $4,8 \%$ en Afrique du Nord, 2,9\% en Afrique de l'Ouest, 3,7\% dans le ProcheOrient. Partout ailleurs, elle représente moins de $1 \%$ du total des viandes rouges.

En dépit de ces faibles contributions, il est notable que la croissance de cette production est plus élevée que celle du bœuf, du mouton et du cheval. Sur la base d'un index 100 en 1961, l'index de production de viande par espèce en 2010 est, dans l'ordre, 448 pour la viande de chèvre, 309 pour le buffle, 285 pour les camélidés, 223 pour le bœuf, 165 pour le mouton et 136 pour les équidés (Faye et Bonnet 2012). Ces tendances sont observées localement par exemple au Tchad où la consommation de la viande de chameau a cru trois fois plus vite que celle des bovins sur le marché de N'Djamena (Koussou et Amine 2012). Il convient toutefois de rappeler ici qu'une part sans doute non négligeable des abattages échappe aux statistiques officielles et qu'en conséquence, la contribution des grands camélidés à la production mondiale de viande rouge est mésestimée. Cependant, l'espèce cameline n'étant pas la seule à faire l'objet d'abattages non déclarés (la proportion chez les petits ruminants est probablement bien plus élevée), il est difficile d'ajuster ces chiffres.

Le poids moyen des carcasses de chameau n'étant passé que de 180 à $200 \mathrm{~kg}$ entre 1961 et 2010 , il est possible qu'il y ait une faible amélioration de la productivité ou une augmentation de l'âge d'abattage. En tout état de cause, la principale raison de la croissance observée pour la viande de chameau est liée à une augmentation du taux d'abattage sur une population en croissance démographique régulière et, peut-être, à une légère augmentation du poids des carcasses.

\section{3 / Les flux marchands et les systèmes de production}

Les pays tels que l'Egypte, l'Arabie saoudite, le Bahreïn, les Emirats Arabes Unis (EAU), la Libye, le Koweït, le sultanat d'Oman et le Maroc (hors provinces sahariennes) sont les principaux pays importateurs d'animaux sur pied destinés à l'abattage, ce qui explique que les taux d'abattage dépassent $20 \%$. Les pays exportateurs sont en majorité situés dans la zone sahélienne. Les pays de la Corne de l'Afrique (Somalie, Ethiopie, Soudan, Djibouti et même Kenya) exportent des dromadaires essentiellement vers la Péninsule arabique et vers l'Egypte. Le Tchad est un pays fournisseur de l'Egypte 
(via le Soudan) et surtout de la Libye. Le Niger approvisionne la Libye et l'Algérie ; le Mali et la Mauritanie exportent vers l'Algérie et le Maroc. Mais le cœur du marché de la viande de chameau se situe dans la Corne de l'Afrique. Sur une population cameline de près de 12 millions de têtes, moins de $4,3 \%$ de ce stock est abattu pour les besoins de consommation locaux. Les données officielles ne donnent pas plus de 41000 animaux exportés par an. Il y a donc indiscutablement un fossé entre ces données et le potentiel réel de production de viande assuré par les exportations dont une partie seulement passe par les circuits autorisés (Alary et Faye 2009).

D'après une enquête réalisée en 2007 dans la région Somali de l'Ethiopie, les exportations ont été estimées pour cette seule région à 37000 têtes avec une marge bénéficiaire de 33 US\$/tête, soit une marge supérieure à celle obtenue avec des petits ruminants ou des bovins (Alary et Faye 2009). Au niveau régional, les exportations « officielles » ne représenteraient que $10 \%$ du total des exportations d'animaux vivants. Ce fossé entre les flux commerciaux déclarés et les flux informels pose un réel problème dans le développement de la filière « export» pour la viande caméline, et ceci explique pourquoi les décideurs se représentent le dromadaire comme un emblème des pasteurs, plutôt que comme une source de revenus pour l'économie locale.

Très florissant au XIX $^{\text {ème }}$ et au $X^{\text {ème }}$ siècle, jusque dans les années 1970, le commerce régional de dromadaires partant des ports somaliens pour l'abattage a été perturbé par les conflits qui ont émaillé la zone depuis la guerre somaloéthiopienne de 1977. Puis, d'autres pays compétiteurs se sont positionnés sur le marché comme l'Australie, le Soudan ou 1'Ethiopie. Des pays tels que Djibouti, le Soudan, voire l'Erythrée qui ont des débouchés maritimes, sont devenus des plaques tournantes de ce commerce aux dépens des ports somaliens. Commerce basé sur les liens tribaux des acteurs de la filière et la confiance entre eux (via des contrats oraux), il a aussi souffert de l'embargo sanitaire de l'Arabie Saoudite suite aux épizooties de Fièvre de la Vallée du Rift (Pratt et al 2005).

En Afrique de 1'Ouest, les flux marchands vont du sud (pays sahéliens) vers le nord (pays du bassin méditerranéen), soit un circuit inverse de celui du bétail bovin qui s'exporte vers les pays côtiers de l'Afrique tropicale. Les réseaux sont informels en plus grande proportion que dans la Corne de l'Afrique. Il est donc malaisé de quantifier l'importance des flux marchands de dromadaires sur pied destinés au marché de la viande.
En Asie, si on excepte le cas particulier de l'Inde avec sa tradition végétarienne (Bénard et al 2008), le commerce de la viande de chameau demeure local, même si la demande récente pour la restauration collective dans les complexes industriels, en particulier en Asie Centrale, offre un débouché rémunérateur. Les filières sont donc courtes et ne débouchent pas sur des flux d'exportation. La viande de Bactriane est en général plus appréciée que celle du dromadaire. Notons toutefois la particularité du Turkménistan, dont la population caméline est composée de dromadaires de race Arvana qui produit une viande recherchée par les consommateurs locaux.

Globalement, les systèmes de production sont peu organisés pour produire une viande de qualité. Souvent les consommateurs considèrent la viande de chameau comme de mauvaise qualité car dans les systèmes traditionnels, les animaux sont généralement abattus tardivement (chamelles et mâles reproducteurs de réforme). Seuls les jeunes mâles de moins de deux ans non conservés pour la reproduction sont destinés à l'abattage. Cependant, il existe une tradition (efficace) d'embouche caméline dans les systèmes pastoraux extensifs de la Corne de l'Afrique visant à fournir sur le marché à l'export des animaux engraissés adultes (4-5 ans). Dans la Péninsule arabique, les consommateurs recherchant plutôt de jeunes animaux de moins de 6 mois à 1 an, la proportion des jeunes mâles dans les abattages y est plus importante. Ces animaux sont majo-

Figure 6. Dépeçage d'un dromadaire à l'abattoir (a) et suspension de la carcasse de dromadaire après dépeçage (b) (photos B. Faye).
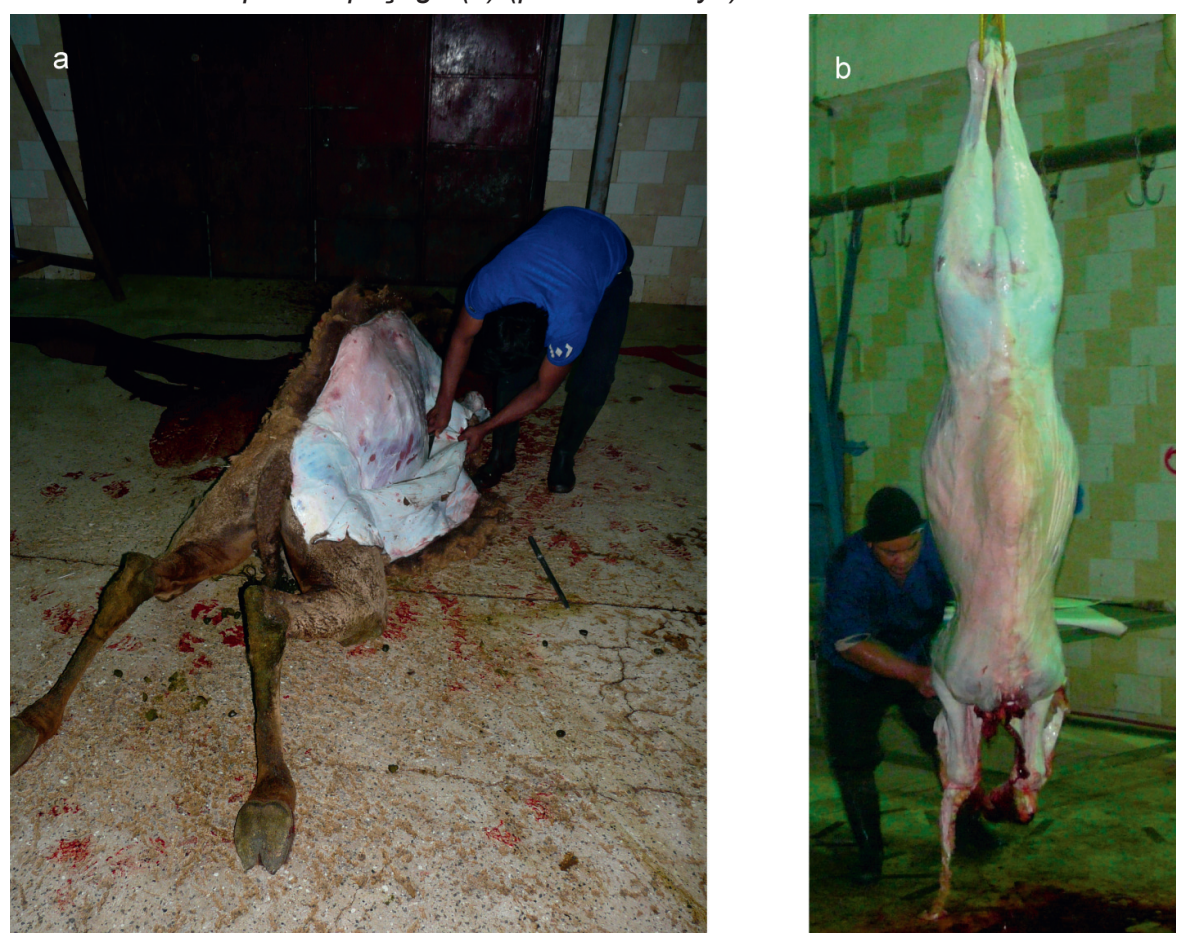
Figure 7. Découpe de la carcasse de dromadaire selon Abouheif et al (1990). La ligne rouge sur la photo 7 a marque la séparation entre quartier avant et quartier arrière et sur la photo $7 \mathrm{~b}$ entre la tête et le cou (photos B. Faye).
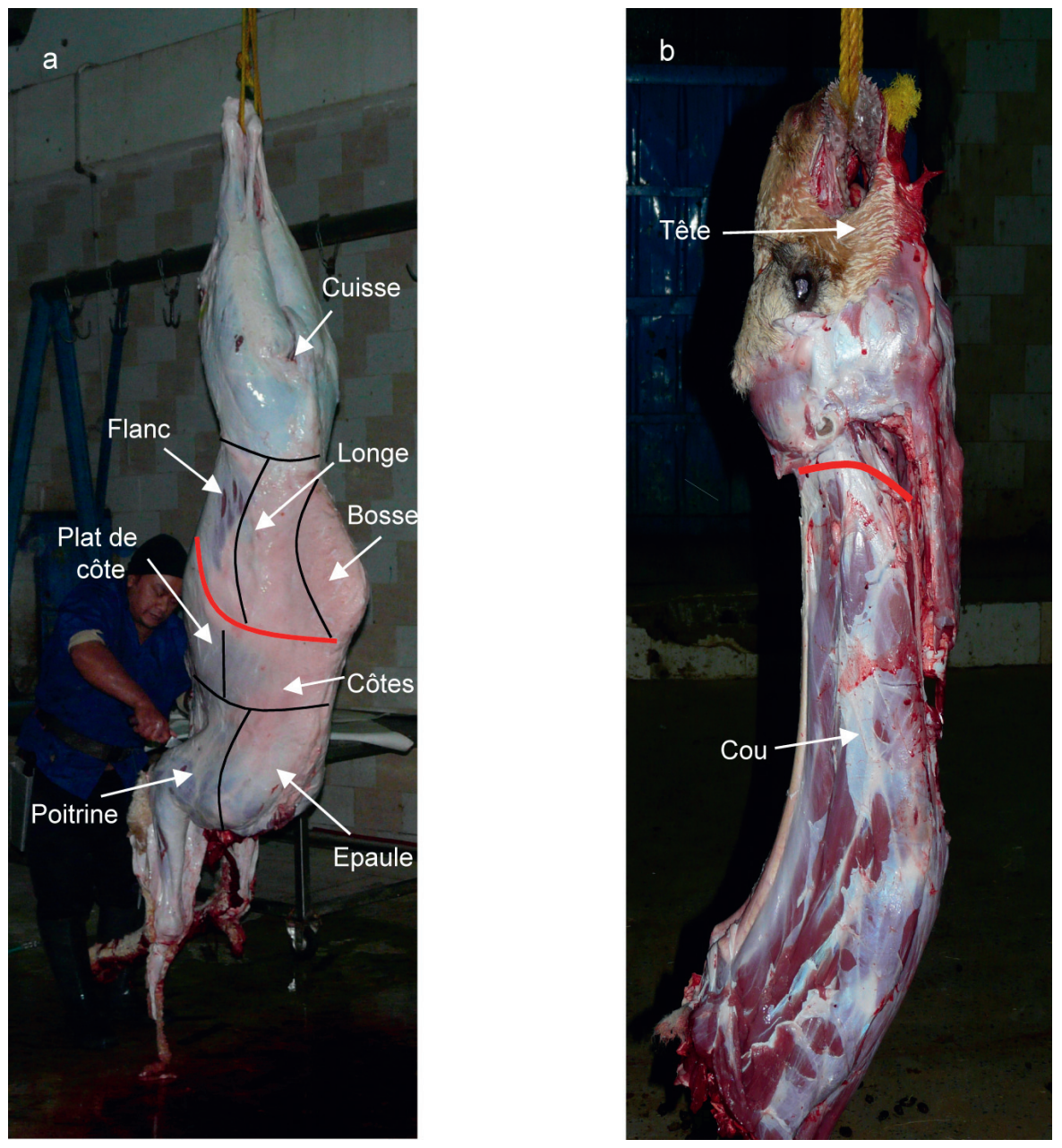

maintenue par un ou plusieurs aides. Dans les conditions traditionnelles, par exemple au Soudan, l'animal est parfois assommé avant la saignée, puis les procédures classiques sont mises en œuvre pour permettre une certification Halal (tête en direction La Mecque, abatteur agrémenté par un imam, louange à Dieu au moment de la saignée) dans les pays musulmans. Le dromadaire est la seule espèce domestique à être dépecée traditionnellement par le dos, la présence de la bosse ne permettant pas de stabiliser la carcasse en position dorsale (Faye 1997). Traditionnellement, l'abattage a lieu au sol, la peau étalée sous l'animal en position ventrale jouant le rôle de protection. Le cou est ensuite ôté et mis à part ainsi que la bosse et les pieds. Les épaules sont ensuite dégagées, et les côtes coupées de la partie vertébrale. Après éviscération et séparation de la colonne vertébrale, les membres postérieurs sont retirés en tranchant le bassin en son milieu (Ulmer et Fisher 2004). Dans les abattoirs modernes, cependant, le dépeçage se fait habituellement sur l'animal suspendu et l'ensemble des opérations se fait dans cette position (figure 6).
Chez un animal adulte, ces parties consommables représentent de 15 à $25 \mathrm{~kg}$ de matière première riche en protéines, vitamines $\mathrm{B}$ et éléments trace (Kadim et al 2013b). Lors de l'abattage traditionnel, il est courant d'offrir un morceau de foie ou de bosse cru à l'abatteur et aux hôtes de passage.

\section{4 / La productivité en viande des grands camélidés}

Les grands camélidés sont des espèces à croissance lente, le poids maximal étant atteint vers 7-8 ans (Tandon et al 1988). D'après les données de la littérature, compilées dans quelques études de synthèses (Richard 1985, Faye 1997 , Kadim et al 2008), le chamelon pèse à la naissance entre 26 et $42 \mathrm{~kg}$ (en moyenne, $35 \mathrm{~kg}$ ) en fonction des races et des conditions d'élevage et sa croissance pondérale varie entre 190 et $350 \mathrm{~g} / \mathrm{j}$ au cours de la première année.

Dans des conditions d'élevage intensif (animaux hors-sol, fourrages irrigués, complémentation en énergie et en protéines), le gain moyen quotidien peut atteindre 450 à $600 \mathrm{~g} / \mathrm{j}$ voire plus en station expérimentale (Richard 1985, Kamoun 1995, Faye 1997). Entre un et deux ans, la croissance peut atteindre et dépasser aisément les $500 \mathrm{~g} / \mathrm{j}$, puis environ $200 \mathrm{~g} / \mathrm{j}$ au-delà de 2 ans, le poids adulte étant atteint vers 7 ans, avec une variation de plus ou moins une année selon les races et les conditions d'élevage. Le poids adulte varie entre 350 et $700 \mathrm{~kg}$ selon le type et le sexe des animaux, mais des individus encore plus lourds sont répertoriés (Saparov et Annageldiyev 2005, Kadim et al 2008).

\section{1 / Caractéristiques de la car- casse de chameau}

Une des particularités de la carcasse de chameau est la présence de la bosse où se concentre l'essentiel des réserves adipeuses (en moyenne 75 à 85\%). La bosse, dont le poids varie entre 15 et $40 \mathrm{~kg}$ (Kamoun 1995), avec des valeurs extrêmes allant de 3 à près de $100 \mathrm{~kg}$, peut représenter une part importante de la carcasse : de 8 à $25 \%$ selon l'état d'embonpoint (Kamoun 1995, Kamili et al 2006), mais aussi selon les races (Faye et al 2001) et donc, le rendement en carcasse en dépend de façon considérable. En règle générale, les données disponibles dans la littérature, précisent si le rendement en carcasse inclut ou non la bosse.

Bosse incluse, le rendement en carcasse varie également en fonction du type et de l'âge des animaux abattus, mais il se situe dans une fourchette 
allant de 47 à $62 \%$ avec une moyenne de 52\% (Kadim et Maghoub 2013). Dans la Corne de l'Afrique, sur des animaux embouchés, des rendements de 59\% sont rapportés (Faye 1997). Dans les mêmes conditions d'élevage, on considère que le rendement en carcasse des dromadaires est supérieur de 1,5 à $2,5 \%$ à celui des bovins. En conséquence, le poids des carcasses varie entre 125 et $300 \mathrm{~kg}$, mais il peut atteindre $400 \mathrm{~kg}$ chez les animaux lourds de la région Somali (Hermann et Fisher 2004) et surtout chez les dromadaires d'Asie et le chameau de Bactriane, en général plus lourds (Kadim et al 2008).

La proportion de muscle dans la carcasse de chameau est comparable à celle des bovins (Mahgoub et al 1995). En revanche, le rapport muscle/os est plus faible que chez les bovins du fait de la taille du squelette de l'animal. Ce rapport est d'environ 3,0 chez les dromadaires du Soudan (Yousif et Babiker 1989). Pour $100 \mathrm{~kg}$ de carcasse (sans bosse), les muscles comptent pour $57 \mathrm{~kg}$, les os pour $25,5 \mathrm{~kg}$ et le gras pour $16,9 \mathrm{~kg}$ (Kamoun 1995). Selon d'autres sources, les proportions sont respectivement de 56, 19 et $13,7 \mathrm{~kg}$ (Yousif et Babiker 1989). La faible proportion de gras intra et intermusculaire est remarquable et représente une caractéristique essentielle de la viande de chameau (Kadim et al 2008).

Le rapport quartier avant/quartier arrière est de 1,61 chez le mâle pour 1,27 chez la femelle, du fait de la plus forte proportion du cou, des épaules et de la bosse chez les premiers (Yousif et Babiker 1989, Kadim et al 2008). La prépondérance du quartier avant (contrairement aux bovins) est en grande partie liée à la présence de la bosse, mais même sans celle-ci, le quartier avant représente $23,8 \%$ du poids vif vs $21,3 \%$ pour le quartier arrière chez le dromadaire Somali (Herrmann et Fischer 2004). Par rapport au poids de la carcasse, les proportions sont respectivement de 44,3,39,7, 7,1 et 8,6\% pour le quartier avant, le quartier arrière, le cou et la bosse (Herrmann et Fischer 2004).

\section{2 / Composition chimique et qualité nutritionnelle}

Au-delà de la variabilité observée selon les races ou types d'animaux, l'âge, le sexe et les conditions d'élevage, la composition de la viande de chameau a été assez bien étudiée dans différents pays et on peut dès lors faire état d'une composition moyenne. Ainsi, la teneur en eau (70 à 77\%) est comparable à celle des autres espèces de rente (Kadim et al 2008, Abdelhadi et al 2012), contrairement à ce que l'on pourrait croire d'une espèce réputée pour son adaptation à la sécheresse. Le dromadaire est également une source appréciable de protéines, sa viande en contenant entre 20 et $23 \%$ selon certaines sources (Al-Owaimer 2000, Kadim et al 2008), un peu moins (de l'ordre de 17\%) selon d'autres auteurs (Abdelhadi et al 2012). La viande de Bactriane contient entre 17 et $18 \%$ de protéines, soit un peu moins que le dromadaire (Raiymbeck et al 2012). Concernant la composition en acides aminés, la viande de chameau contient une relative faible proportion d'histidine, de valine, de leucine, d'isoleucine et tout particulièrement de tryptophane, ce qui la rapproche, de ce point de vue, de la viande d'agneau (Kadim et al 2008), mais les différences sont peu marquées avec les autres types de viande.

La teneur en matières grasses est bien évidemment très variable en fonction de l'état d'engraissement de l'animal. Dans la littérature, des valeurs varient entre 1,4 et 10\% (Babiker et Yousif 1990, Dawood et Alkanhal 1995, Al-Owaimer 2000, Kadim et al 2006), une tendance à l'augmentation du gras intramusculaire étant observée en fonction de l'âge en même temps que le taux de protéines a tendance à décroître (Abdelhadi et al 2011), comme pour toutes les espèces. La viande des camélidés en général est réputée pour sa faible teneur en cholestérol (tableau 1), ce qui semble être une caractéristique de l'espèce et représente un argument commercial de poids pour affirmer le caractère sain de ce produit (Kadim et al 2008).

Cependant, la variabilité de la teneur en cholestérol du muscle est importante en fonction de différents facteurs liés à l'animal (âge, sexe, facteurs alimentaires...) ou aux méthodes d'analyse. Il convient d'être prudent sur la signification des résultats, mais en tout état de cause, toutes les études comparatives ont confirmé l'avantage de la viande de chameau sur ce point. La valeur "santé " de la viande de chameau serait égale-

Tableau 1. Teneurs moyennes en cholestérol $(\mathrm{mg} / 100 \mathrm{~g})$ dans la viande de différentes espèces (d'après El-Magoli et al 1973, Sinclair et al 1982, Holland et al 1991, Abu-Tarboush et Dawood 1993, Sales 1996, Kadim et al 2008, Madruga et al 2008, Yousefi et al 2012).

\begin{tabular}{|c|c|}
\hline Espèce & Cholestérol \\
\hline Dromadaire & $50-61$ \\
\hline Vache & $59-73$ \\
\hline Chèvre & $63-71$ \\
\hline Mouton & $53-78$ \\
\hline Porc & $60-80$ \\
\hline Poulet & $57-76$ \\
\hline Kangourou & 62 \\
\hline Autruche & 62 \\
\hline
\end{tabular}

ment à prévaloir du fait de sa composition en Acides Gras (AG) (Rawdah et al 1994). En effet, les AG mono-insaturés représentent un tiers du total des AG de la viande et sont dominés par l'acide oléique (C18:1) et l'acide palmitoléique (C16:1). Les AG polyinsaturés sont également en relative abondance $(18,6 \%)$, en particulier l'acide linoléique (C18:2) et l'acide arachidonique (C20:4). Le rapport polyinsaturés/saturés apparaît dès lors favorable $(0,36)$ comparé à celui relevé dans la viande de bœuf $(0,22)$ ou de mouton $(0,26)$ (Sinclair et al 1982). Le rapport linoléique/linolénique est en particulier beaucoup plus élevé dans la viande de chameau $(10,9)$ que chez les autres espèces de rente : 2,0 dans la viande de bœuf, 2,4 dans celle du mouton et 2,8 chez la chèvre (Sinclair et al 1982).

Les minéraux représentent entre 1,1 et $1,5 \%$ de la viande de chameau (AlOwaimer 2000, Kadim et al 2006). Chez le chameau de Bactriane, la teneur dépasse rarement $1 \%$ et le potassium représente la partie la plus importante, entre 37 et $80 \%$ selon les muscles analysés (Raiymbek et al 2013). La composition en minéraux du Bactriane est en fait assez proche de celle des dromadaires (Kadim et al 2006). La teneur en calcium (de l'ordre de $6,5 \mathrm{mg} / 100 \mathrm{~g}$ ) apparaît plus élevée que dans la viande de bœuf, ce qui serait dû à la structure plus serrée de la viande de chameau (Kadim et al 2008). On dispose aussi de quelques données concernant la teneur en vitamines (Ulmer et al 2004) : 0,12 $\mathrm{mg} / 100 \mathrm{~g}$ pour la thiamine (B1), 0,18 pour la riboflavine (B2), 0,25 pour la pyridoxine (B6) et 0,61 pour l' $\alpha$-tocopherol (vitamine $\mathrm{E})$.

Il y a une variation de cette composition chimique selon la localisation musculaire (Herrmann et Fischer 2004). Par exemple, les muscles de l'épaule ont des teneurs en protéines significativement plus élevées $(77-78 \%$ de la MS) et en minéraux plus faibles $(1,1 \%$ de la MS) 
que ceux de la longe (73 et $0,6 \%$ respectivement). Les acides aminés libres et les minéraux inorganiques sont en proportion plus élevée que dans la viande de bœuf, ce qui serait lié à la faible teneur en matières grasses de la viande de chameau (Kadim et Mahgoub 2006).

\section{5 / Les qualités sensorielles de la viande de chameau}

La viande de chameau est qualifiée de couleur " rouge framboise " et parfois brune chez les animaux plus âgés (du fait d'une plus forte concentration de myoglobine) avec un léger goût sucré qui serait dû à une relative richesse en glycogène (Kadim et al 2008). Le gras de la viande est, quant à lui, de couleur très blanche. Les consommateurs reprochent souvent à la viande de dromadaire, son manque de tendreté qui est le plus souvent lié au fait que nombre d'animaux abattus dépassent les 10 ans. Or, la force de cisaillement (indicateur de dureté) chez un chameau de 6 à 8 ans est plus élevée de 40 et $48 \%$ comparée à un animal de 3 à 5 ans et à un animal de 1 à 3 ans respectivement (tableau 2 ). De la même manière, la perte en eau à la cuisson est d'autant plus faible que la viande provient d'un animal âgé (tableau 2) comme chez toutes les espèces. Bien évidemment, la viande est moins tendre et la palatabilité diminue avec l'âge (Kadim et al 2006). Il apparaît d'après ces résultats que l'âge optimum de l'abattage se situe entre 1 et 3 ans, ce qui correspond aux pratiques pour les jeunes mâles.

Le $\mathrm{pH}$ ultime, déterminé par la déplétion du glycogène et l'accumulation de l'acide lactique, varie entre 5,7 et 6,0 chez le dromadaire (Kadim et al 2006) et le chameau de Bactriane (Raiymbek et al $2012 \mathrm{a}$ et b), il a tendance à diminuer avec l'âge de l'abattage comme pour les autres espèces. Dans les zones pastorales où l'abattage communautaire concerne le plus souvent des animaux de réforme, et où il n'y a pas de technique de maturation de la viande, la pratique la plus courante est de consommer la viande de dromadaire après l'avoir enveloppée dans des feuilles de papayer pendant 24 à $48 \mathrm{~h}$. Ces feuilles contiennent de la papaïne aux vertus protéolytiques qui attendrissent la viande (Boudry 2007).

Pour les jeunes mâles, les caractéristiques qualitatives générales de la viande de chameau sont très proches de celles du bœuf. Au demeurant, il est difficile pour un consommateur non-averti de faire la différence. La force de cisaillement (" shear force ») est comparable entre le muscle longissimus d'un cha-
Tableau 2. Effet de l'âge sur les caractères qualitatifs du muscle Longissimus thoracis chez le chameau (d'après Kadim et al 2006).

\begin{tabular}{|l|c|c|c|}
\hline \multirow{2}{*}{} & \multicolumn{3}{|c|}{ Groupe d'âge (années) } \\
\cline { 2 - 4 } & $1-3$ & $3-5$ & $5-8$ \\
\hline $\mathrm{pH}$ ultime & 5,91 & 5,84 & $5,71^{*}$ \\
\hline Force de cisaillement $(\mathrm{N})$ & 6,84 & 7,95 & $13,19^{*}$ \\
\hline Longueur des sarcomères $(\mu \mathrm{m})$ & 1,85 & 1,24 & $1,06^{*}$ \\
\hline Index de fragmentation des myofibrilles $(\%)$ & 80,99 & 73,3 & $60,4^{*}$ \\
\hline Perte en eau à la cuisson $(\%)$ & 26,06 & 23,72 & $22,42^{*}$ \\
\hline
\end{tabular}

${ }^{*} \mathrm{P}<0,05$

meau de 2-4 ans $(6,98)$ et celui d'un bœuf de 2-3 ans $(6,45)$. La longueur des sarcomères est respectivement de 1,89 et $1,83 \mu \mathrm{m}$ (Kadim et al 2008). Les paramètres de couleur sont également très proches : concernant respectivement les indices de couleurs, luminosité $\left(\mathrm{L}^{*}\right)$, rouge $\left(\mathrm{a}^{*}\right)$ et jaune $\left(\mathrm{b}^{*}\right)$, les valeurs sont $31,7 / 16,2 / 7,3$ chez le chameau et de 33,6/18,2/6,4 chez le bœuf (Kadim et Maghoub 2006). Toutefois, il existe là aussi des variations selon les auteurs qui analysent des viandes d'animaux élevés dans des contextes différents sans toutefois préciser les conditions d'élevage et en particulier d'alimentation, la plupart des prélèvements étant réalisés à l'abattoir sur des animaux sans commémoratifs précis. Par exemple, concernant les paramètres de couleur, Abdelhadi et al (2012) rapportent des valeurs de 34,0 ( $\left.\mathrm{L}^{*}\right), 13,2$ ( $\mathrm{a}^{*}$ ) et 11,2 (b*) sur des dromadaires du Soudan avec un effet saisonnier lié à l'alimentation des animaux variant au cours de l'année.

Les caractéristiques contractiles de la viande de chameau ont été également explorées et il s'avère que seules deux isoformes de chaîne lourde de myosine (MyHC I et MyHC IIa) sont détectables par électrophorèse dans le muscle de chameau, mais que les isoformes IIx et IIb caractérisant les fibres rapides glycolytiques et décrites dans la viande de bœuf, ne sont pas observées dans celle du chameau, du moins dans le muscle Longissimus Thoracis (LT) (Abdelhadi et al 2012). Concernant le type de fibres dans le muscle de chameau, les fibres I prédominent $(67,8 \%)$ par rapport aux fibres IIa $(32,2 \%)$. Ce rapport ainsi que l'activité métabolique du muscle varient au cours de l'année en fonction des saisons (Abdelhadi et al 2012). Un rapport différent est observé par d'autres auteurs, avec une prédominance des fibres de type IIa (40,1 à 46,7\% selon les références) et la présence de fibres IIb $(29,9$ à $38,8 \%$ ) détectées par des techniques histochimiques, et sur des animaux n'ayant pas les mêmes âges (Kassem et al 2004, Kadim et al 2009).

Comme chez le bovin, la contribution des caractéristiques biochimiques du muscle à la variabilité de la tendreté est faible. Toutefois, il apparaît que les muscles les plus glycolytiques tendent à être plus durs chez le chameau (Hocquette et al résultats non publiés) comme chez le bovin (Chriki et al 2012). Il existe très peu d'informations en revanche sur le collagène comme le soulignent Kadim et al (2013c).

Si la qualité de la viande de dromadaire a été évaluée jusqu'à présent selon les méthodes classiques utilisées pour les autres espèces de rente, les nouvelles technologies mises en cuvre pour la viande de bovin, sont envisagées aussi pour évaluer la viande de chameau : sondes optiques pour évaluer le gras sous-cutané ou le gras musculaire, spectrophotométrie par fibre optique pour mesurer la concentration en myoglobine ou la teneur en carotène, sonde électromécanique pour évaluer la tendreté de la viande, analyse d'image vidéo pour apprécier la muscularité d'une carcasse ou encore réflectomètre de surface pour déterminer le contenu en collagène ou en triglycérides, sont quelques unes de ces nouvelles technologies parfaitement applicables à la viande des camélidés (Swatland 2012). De même, la recherche de marqueurs génomiques prédicteurs de la tendreté (Hocquette et al 2012) est en cours de réalisation.

\section{6 / La valeur nutritive de la viande de dromadaire et des produits carnés}

Une carcasse moyenne d'environ $210 \mathrm{~kg}$ fournit en moyenne $35,2 \mathrm{~kg}$ de protéines et $997312 \mathrm{KJ}$ d'énergie, soit respectivement $16,7 \mathrm{~g}$ et $47490 \mathrm{KJ}$ pour $100 \mathrm{~g}$ de viande. En théorie, une carcasse de dromadaire assure les besoins énergétiques d'un homme adulte pour 5 jours et les besoins en protéines pour 35 jours (Faye 1997).

La viande de dromadaire est rarement transformée. Cependant, séchage, saumurage et fumage sont des pratiques traditionnelles courantes. Dans la Corne de l'Afrique, par exemple, les pasteurs, qui sacrifient un animal pour l'autocon- 
Figure 8. Saucisses de chameau dans les échoppes de l'Est de la Turquie (photo F. Brey).

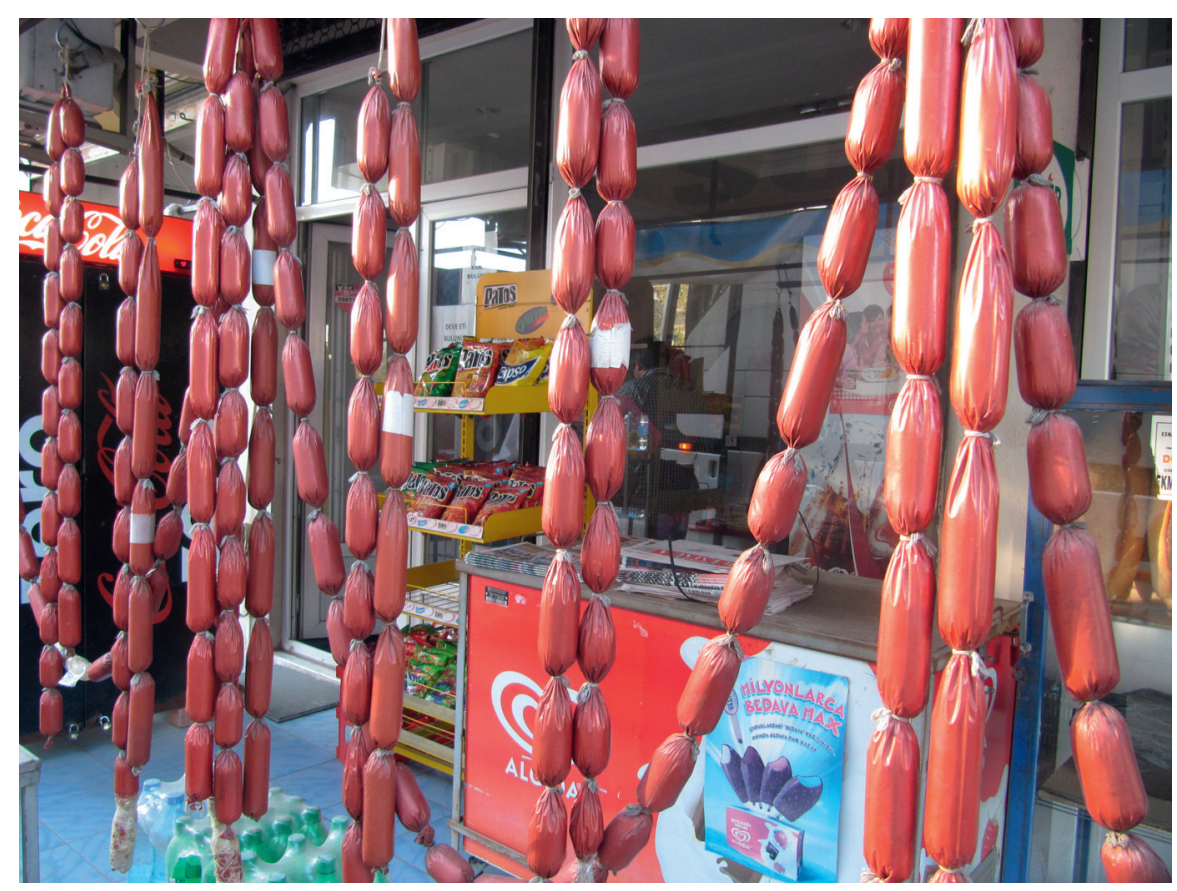

sommation ne pouvant pas utiliser la totalité de la carcasse dans un délai suffisamment bref, pratiquent couramment le séchage solaire. Ils obtiennent une viande boucanée, additionnée d'épices (le kounta des Afar) qui peut se conserver plusieurs mois et qui est appréciée des caravaniers lors de leurs déplacements. Des sachets de viande de chameau séchée sont proposés également dans les supermarchés (Ulmer et al 2004). Des préparations telles que les saucisses ou les " camelburger " sont proposées aujourd'hui dans les commerces urbains (figure 8). Des technologies modernes sont notamment mises en œuvre dans certains pays pour préparer des «burgers » et des saucisses à partir de viande de chameau (Mansour et Ahmed 2000). Les produits obtenus ont une composition chimique comparable à ceux fabriqués à partir de la viande de bœuf, avec cependant un taux plus élevé d'humidité $(73,6 \%)$ et de miné$\operatorname{raux}(4,1 \%)$.

Plusieurs tests s'appuyant sur des panels de dégustateurs ont été expérimentés, soit pour évaluer les qualités physiques et sensorielles de la viande de chameau en fonction de sa teneur en matière grasse ou de son statut de viande fraîche ou congelée (Dawood 1995), soit petits ruminants. L'efficacité alimentaire du dromadaire est en effet assez faible : entre 8,5 et $9,5 \mathrm{~kg}$ de MS ingérée par $\mathrm{kg}$ de gain de poids vif (Kamoun 1995).

En dépit de cette faible productivité, la production bouchère caméline devrait connaître un essor incontestable et ce pour plusieurs raisons. Tout d'abord, le prix de la viande de chameau est souvent inférieur à celui des bovins et des ovins, en tout cas sur les marchés les plus importants comme celui du Caire en Egypte, du fait de coûts de transaction plus faibles et de circuits comprenant moins d'intermédiaires, pour une production par ailleurs encore très extensive et donc avec peu d'intrants. Ceci permet donc un accès aux protéines carnées pour les populations souvent les plus défavorisées et assure à la viande caméline une certaine compétitivité. Par ailleurs, la bonne tenue de l'espèce lors des sécheresses a permis un transfert partiel mais notable de la demande en viande bovine (produit de prix très variable du fait des déstockages massifs lors des sécheresses saisonnières ou exceptionnelles) vers la viande caméline (produit moins victime de la détérioration des termes de l'échange pastoral en cas de sécheresse, car moins soumis aux déstockages massifs lorsque le prix des céréales devient élevé). Ce phénomène, patent dans des pays sahéliens (Niger, Tchad, nord-Nigéria), a beaucoup joué dans la restructuration des populations animales domestiques (substitution des bovins par des camelins dans les troupeaux sahéliens), mais a contribué aussi à renforcer le poids commercial de l'espèce dromadaire dans l'alimentation auprès des consommateurs. Cependant, dans les pays du Golfe où l'intensification de la production cameline est à l'œuvre, on devrait assister à une autre évolution du marché de la viande, plus proche des circuits existants pour l'espèce bovine. En tout état de cause, le potentiel de production de viande par l'espèce cameline est tout à fait intéressant pour la satisfaction des besoins en protéines animales des consommateurs des pays arides, et ce d'autant plus qu'on lui prête, du fait de sa faible teneur en cholestérol et de sa bonne tenue en protéines et en minéraux, des vertus diététiques incontestables. 


\section{Références}

Abdallah H.R., Faye B., 2013.Typology of camel farming system in Saudi Arabia. Emir J. Food Agric., 25, 250-260

Abdelhadi O., Babiker S.A., Faye B., Kijora C., 2011. Effect of ageing time on chemical composition and quality of the desert camel meat (Camelus dromedarius). Proc. Tropentag. "Development on the margin", Bonn, Oct 5-7, Germany, poster ID410.

Abdelhadi O., Babiker S.A., Picard B., Jurie C., Jailler R., Hocquette J.F., Faye B., 2012 Effect of season on contractile and metabolic properties of desert camel muscle (Camelus dromedarius). Meat Sci., 90, 139-144.

Abouheif M.A., Basmaeil S.M., Bakkar M.N., 1990. A standard method for jointing camel carcasses with reference to the effect of slaughter age on carcass characteristics in Najadi Camels. I. Wholesale cut weight. Asian Austral. J. Anim. Sci., 3, 97-102.

Abu-Tarboush H.M., Dawood A., 1993. Cholesterol and fat contents of animal adipose tissues. Food chemist., 46, 89-93.

Alary V., Faye B., 2009. Overview of the camel chains in East of Africa: importance of gaps between the data and the apparent reality. Proc. $2^{\text {nd }}$ Conf. ISOCARD, Djerba, Tunisia, 12 14 march 2009, abstr. 92, 74

Al-Bachir M., Zeinou R., 2009. Effect of gamma irradiation on microbial load and quality characteristics of minced camel meat. Meat Sci., 82, 119-124.

Al-Owaimer A.N., 2000. Effect of dietary Halophyte Salicornia bigelovii Torr on carcass characteristics, minerals, fatty acids and amino acids profile of camel meat. J. Appl. Anim. Res., 18, 185-192.

Babiker S.A., Yousif K.H., 1990. Chemica composition and quality of camel meat. Meat Sci., 27, 283-287.

Bekhit A.E., Farouk M.M., 2013. Nutritive and health value of camel meat. In: "Camel meat and meat products", Kadim I., Maghoub O., Faye B., Farouk M. (Eds). CABI, Oxfordshire, UK, 205-223

Benard C., Faye B., Moulin C.H., KohlerRollefson I., 2008. A typology of the camel keepers in the Jaisalmer district, Rajasthan, India, J. Camel Pract. Res., 15, 231-238.

Boudry I., 2007. Les formulations topiques à base d'enzymes. Ann. Pharm. Franç., 65, 142-144.

Cabalion S., 2013. Le système « domesticatoire » touareg (Tagaraygarayt, Niger). Thèse Ecole des Hautes Etudes en Sciences Sociales, Paris, France, $515 \mathrm{p}$.

Chriki S., Gardner G.E., Jurie C., Picard B., Micol D., Brun J.P., Journaux L., Hocquette J.F., 2012. Cluster analysis application in search of muscle biochemical determinants for beef tenderness. BMC Biochem., 13, 29.

Dawood A., 1995. Physical and sensory characteristics of Najdi-camel meat. Meat Sci., 39, 59-69.

Dawood A., Alkanhal M.A., 1995. Nutrient composition of Najdi-Camel Meat. Meat Sci., 39, 71-78.

El-Magoli S.B., Awad A.A., El-Wakeil F.A., 1973. Intramuscular lipid chemistry of beef and camel Longissimus dorsi muscle. Egyptian J. Food Sci., 1, 75-84

\section{FAOSTAT, 2011. http://faostat.fao.org/}

Faye B., 1997. Guide de l'élevage du dromadaire. Ed. Sanofi, Libourne, France, 126p.

Faye B., 2013. Camel meat in the world. In: "Camel meat and meat products", Kadim I. Maghoub O., Faye B., Farouk M. (Eds). CABI, Oxfordshire, UK, 7-16.

Faye B., Bonnet P., 2012. Camel sciences and economy in the world: current situation and perspectives. Proc. $3^{\text {rd }}$ ISOCARD Conf Keynote presentations, Johnson E.H. et al (Eds), 29 ${ }^{\text {th }}$ January - $1^{\text {st }}$ February, Mascate, Sultanate of Oman, 2-15.

Faye B., Porphyre V., 2011. Le dromadaire et le cochon : deux visions opposées de l'élevage ? Nature Sci. Soc., 19, 365-374.

Faye B., Jouany J.P., Chacornac J.P., Ratovonanahary M., 1995. L'élevage des grands camélidés. Analyse des initiatives réalisées en France. INRA Prod. Anim., 8, 3-17.

Faye B., Bengoumi M., Messad S., Chilliard Y., 2001. Fat storage and adipocyte patterns in camel: a tool for management of reproduction. Adv. Reprod., 5, issue 3, 10c

Faye B., Grech S., Korchani T., 2002. Le dromadaire, entre féralisation et intensification. Anthropozoologica, 39, 7-13.

Herrmann K., Fischer A., 2004. Method for hygienic slaughter of camels. In: Farah Z., Fisher A. (Eds). Milk and meat from the camel. Handbook on products and processing. vdf Hochschulverlag AG an der ETH Zurich, Zurich/Singen, 89-108.

Hocquette J.F., Bernard-Capel C., Vidal V., Jesson B., Levéziel H., Cassar-Malek I., 2012. The GENOTEND chip: a new tool to analyse gene expression in muscles of beef cattle for beef quality prediction. BMC Vet. Res., 8, 135.

Holland B., Welch A.A., Unwin I.D., Buss D.H., Paul A.A., Southgate D.A.T., 1991 McCance and Widdowson's the composition of foods, $6^{\text {th }}$ and extended Ed. Bungay, Suffolk, UK: The Royal Society of Chemistry and Ministry of Agriculture, Fisheries and Food, Richard Clay Ltd.

Kadim I.T., Mahgoub O., 2006. Meat quality and composition of Longissimus thoracis from Arabian camel (Camelus dromedaries) and Omani beef: A comparative study. In Proceedings of the $1^{\text {st }}$ conference of the international society of camelids research and development (ISOCARD), Al-Ain, United Arab Emirates, p118.

Kadim I.T., Mahgoub O., Al-Marzooqi W. Al-Zadgali S., Annamali K., Mansour M.H., 2006. Effects of age on composition and quality of muscle Longissimus thoracis of the Oman Arabian camel (Camelus dromedaries). Meat Sci., 73, 619-625.

Kadim I.T., Mahgoub O., Purchas R.W. 2008. A review of the growth, and of the carcass and meat quality characteristics of the one-humped camel (Camelus dromedaries). Meat Sci., 80, 555-569.

Kadim I.T, Al-Hosni Y, Mahgoub O., AlMarzooqi W., Khalaf S.K., Al-Maqbaly R.S 2009. Effect of low voltage electrical stimulation on biochemical and quality characteristics of Longissimus thoracis muscle from onehumped camel (Camelus dromedaries). Meat Sci., $82,77-85$.
Kadim I.T, Maghoub O, 2013. Camel carcass quality. In: "Camel meat and meat products", Kadim I., Maghoub O., Faye B., Farouk M. (Eds). CABI, Oxfordshire, UK, 98-112.

Kadim I.T., Mahgoub O., Faye B., Farouk M., 2013a. Camel meat and meat products, CABI, Oxfordshire, UK, 258p.

Kadim I.T., Farouk M., Maghoub O., Bekhit A., 2013b. Slaughtering and processing of camels. In: "Camel meat and meat products", Kadim I., Maghoub O., Faye B., Farouk M. (Eds). CABI, Oxfordshire, UK, 55-72.

Kadim I.T., Al-Karousi A., Mahgoub O., AlMarzooqi W., Khalaf S.K., Al-Maqbali R.S., Al-Sinani S.H., Raiymbek G., 2013c. Chemical composition, quality and histochemical characteristics of individual dromedary camel (Camelus dromedarius) muscles. Meat Sci. 93, 564-571.

Kamili A., Bengoumi M., Faye B., 2006. Assessment of body condition and body composition in camel by barymetric measurements. J. Camel Pract. Res., 13, 67-72.

Kamoun M., 1995. Dromedary meat: production, qualitative aspects and acceptability for transformation. Option Méditerranéennes Série B, Etudes et Recherches, 13, 105-130.

Kassem M.T., El-Sayed M.T., Ahmed A., 2004. Micostructural characteristics of Arabian camel meat. J. Camel Sci., 1, 86-95.

Khorchani T., Hammadi M., Moslah M., 2005. Artificial nursing of camel calves: an effective technique for calves safeguard and improving herd productivity. In: Faye B., Esenov P. (Eds). Proc. Int. Workshop, «Desertification combat and food safety: the added value of camel producers», Ashkabad (Turkmenistan), 19-22 april 2004. In: Vol. 362 NATO Sciences Series, Life Behav. Sci. IOS press Publ., Amsterdam, The Netherlands, 177-182.

Koussou M.O., Amine M.A., 2012. Le commerce de viande de dromadaire : un nouveau débouché pour les éleveurs sahéliens ? J Anim. Plant Sci., 16, 2312-2330.

Madruga M.S., Torres T.S., Carvalho F.F., Queiroga R.C., Narain N., Garrutti D., Souza M.A., Carla N., Mattos W., Costa R.G., 2008. Meat quality of Moxotó and Canindé goats as affected by two levels of feeding. Meat Sci., 80, 1019-1023.

Mahgoub O., Olvey F.H., Jeffrey D.C., 1995. Growth and composition of the Oman Dhofari cattle. 1, Body weight growth and carcass composition. Asian Austral. J. Anim. Sci., 8, 611-616.

Mansour M.E. Ahmed S.M., 2000. Advanced technology in camel meat processing. The Camel Newsletter, 17, 27-29.

Mbaga M., 2013. The economic potential of camel meat. In: "Camel meat and meat products", Kadim I., Maghoub O., Faye B., Farouk M. (Eds). CABI, Oxfordshire, UK, 224-238.

Pratt A.N., Bonnet P., Jabba M., Ehui S., de Haan C., 2005. Benefits and Costs of compliance of sanitary regulations in livestock markets: the case of Rift Valley Fever in the Somali region of Ethiopia, Report International Livestock Research Institute, Nairobi, Kenya, The World Bank. 
Raiymbek G., Faye B., Konuspayeva G., Kadim I.T., 2012. Meat quality characteristics of Infraspinatus, Triceps brachii, Longissimus thoraces, Biceps femoris, Semitendinosus, and Semimembranosus of Bactrian (Camelus Bactrianus) camel muscles. Revue KAZNU, série biologique, 2, 27-32.

Raiymbek G., Faye B., Serikbaeva A., Konuspayeva G., Kadim I.T., 2013. Chemical composition of Infraspinatus, Triceps brachii, Longissimus thoraces, Biceps femoris, Semitendinosus, and Semimembranosus of Bactrian (Camelus Bactrianus) camel muscles. Emirates. J. Food. Agric., 25, 261-266.

Rawdah T.N., El-Faer M.Z., Koreish S.A., 1994. Fatty acid composition of the meat and fat of the one-humped camel (Camelus dromedaries). Meat Sci., 37, 149-155.

Richard D., 1985. Le dromadaire et son élevage. Publ. IEMVT, Coll. "Etudes et synthèses", CIRAD-Montpellier, France, 162p.

Saalfeld W.K., Edwards G.P., 2010. Distribution and abundance of the feral camel (Camelus dromedarius) in Australia. Rangeland J., 32, 1-9.

Sales J., 1996. Histological, biological, physiological and chemical characteristics of different ostrich muscles. J. Sci. Food Agric. 70, 109-114.

Saparov G., Annageldiyev O., 2005. Meat productivity of the camel Arvana breed and ways to increase it. In: Faye B., Esenov P. (Eds). Proc. Int. Workshop, "Desertification combat and food safety: the added value of camel producers", Ashkabad (Turkmenistan), 19-22 april 2004. In: Vol. 362 NATO Sciences Series, Life Behav. Sci., IOS press Publ., Amsterdam, The Netherlands, 224-227.

Schwartz H. Dioli M. 1992. The OneHumped camel in eastern-Africa. Publ. Verlag, Weikersheim, Germany, 282p.

Sinclair A.J., Slattery W.J., O’Dea K., 1982. The analysis of polyunsaturated fatty acids in meat by capillary gas-liquid chromatography. J. Sci. Food Agric., 33, 771-776.

Smits M.G., Montety G.J., 2009. Ammonia emission from camel dairy in the Netherlands. J. Camel Pract. Res., 16, 139-142.

Swatland H.J., 2012. Developing new technology for measuring camelid meat quality., J. Camelid Sci., 5, 25-40.

Tandon S.N., Bissa U.K., Khanna N.D. 1988. Camel meat: Present status and future prospects. Annals of Arid Zone, 27, 23-28.
Ulmer K., Fisher A., 2004. Traditional slaughtering, carcass dressing and processing of camels. In: Farah Z., Fischer A. (Eds). Milk and meat from the camel. Vdf Hochschulverlag AG an der ETH Zurich, ETH Zentrum, Zurich, 85-88.

Ulmer K., Herrmann K., Fischer A., 2004. Meat products from camel meat. In: Farah Z., Fischer A. (Eds). Milk and meat from the camel. Vdf Hochschulverlag AG an der ETH Zurich, ETH Zentrum, Zurich, 137-228.

Ward B., Burrows N., Lethbridge M., 2010. Inland invaders: a million wild camels. Landscope, 26, 40-44.

Yousefi A.R., Kohram H., Shahneh A.Z., Nik-khah A., Campbell A.W., 2012. Comparison of the meat quality and fatty acid composition of traditional fat-tailed (Chall) and tailed (Zel) Iranian sheep breeds. Meat Sci., 92, 417-422.

Yousif O.K., Babiker S.A., 1989. The desert camel as meat animals. Meat Sci., 26, 245254.

Zegeye A., 1999. A note on the influence of heat treatment, salting and smoking on the acceptability of camel products. Meat Sci., 53, 217-219.

\section{Résumé}

La viande de chameau est un produit régulièrement consommé dans les pays arides et c'est un des rares produits de cette espèce faisant l'objet d'un marché d'export régional, voire international si on inclut la viande de chameaux " marrons » d'Australie, bien qu'une part mal évaluée de ce marché se situe dans le secteur informel. Les principaux pays exportateurs se situent dans la Corne de l'Afrique et dans la région sahélienne alors que les pays importateurs sont surtout les pays du Golfe et d'Afrique du Nord. La productivité en viande chez le chameau est plutôt faible bien que les rendements carcasse se rapprochent de ceux des bovins, surtout chez les animaux provenant des systèmes de production plus intensifs. Il existe toutefois une tradition d'embouche cameline pastorale obtenant de bons résultats. La viande de chameau est assez proche de la viande de bœuf tant dans sa composition chimique globale que dans ses particularités gustatives et sa valeur nutritionnelle. Toutefois, du fait de la concentration du gras dans la bosse, la viande de chameau apparaît relativement maigre et particulièrement pauvre en cholestérol, ce qui peut en faire un argument commercial certain.

\section{Abstract}

\section{The production of camel meat: state of knowledge, current situation and prospects}

Camel meat is a product regularly consumed in arid countries and it is one of the rare products from this species being subject to a regional export market, even international, if the meat from Australian wild camel is included although a poorly evaluated part of this market is out of the official sector. The main exporting countries are located in the Horn of Africa and the Sahelian area whereas the importing countries are the Gulf States and North Africa. Meat productivity in the camel is rather low although the dressing percentages approach those of cattle, especially in animals coming from the more intensive production systems. However, there is a tradition of pastoral fattening having good results. Camel meat is rather close to beef as well in its total chemical composition as in its gustatory characteristics and nutritive value. However, because of fat concentration in the hump, camel muscles give relatively low-fat meat and are particularly low in cholesterol, which can make a good commercial argument.

FAYE B., ABDELHADI O., RAIYMBEK G., KADIM I., HOCQUETTE J.-F., 2013. La production de viande de chameau : état des connaissances, situation actuelle et perspectives. INRA Prod. Anim., 26, 3, 289-300. 